Les cellules germinales de tous les animaux possèdent une propriété importante qui se manifeste pendant la vie embryonnaire et la vie adulte : la mobilité. Tout au début $d u$ développement, les cellules germinales se trouvent en dehors des ébauches génitales. Par la suite, elles migrent et pénètrent dans les gonades, où elles se différencient. Arrivées à maturité, les cellules sexuelles recouvrent leur liberté et leur mobilité. Chez beaucoup d'animaux, elles sont guidées à l'extérieur par des conduits spécialisés. Typiquement, la gamétogenèse comporte donc deux phases libres, encadrant une phase captive. Cet article propose une interprétation évolutive des trois phases de la gamétogenèse.

L'hypothèse de base est que les animaux les plus primitifs avaient une lignée germinale bien individualisée, mais ni gonades ni conduits génitaux. Les trois phases de la gamétogenèse sont assimilées à trois étapes successives dans l'évolution du système reproducteur. Dans une première étape, les cellules germinales étaient libres au sein de l'organisme. Dans une deuxième étape, un organe spécialisé - ovaire ou testicule - a capté les cellules sexuelles, les intégrant d'une façon qui permet leur libération ultérieure dans l'organisme, puis au dehors. Dans une troisième étape, les cellules sexuelles mûres, relâchées par la gonade, ont été reprises par des conduits évacuateurs, souvent dérivés du système excréteur, ou se branchant sur lui. Parmi les nombreux processus que comporte la gamétogenèse, certains ont gardé une indépendance totale ou partielle envers les gonades. Il s'agit probablement d'une situation primitive, témoin ou vestige d'un stade évolutif antérieur à l'apparition des glandes génitales.

\title{
Une interprétation évolutive de la gamétogenèse animale
}

\author{
Herman Denis, Jean-Claude Lacroix
}

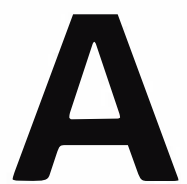
u fil de l'évolution, les animaux ont adopté des stratégies reproductives d'une surprenante variété. Cela se manifeste dans chacun des deux modes de propagation adoptés par les animaux : la reproduction asexuée et la reproduction sexuée. Nous limitons ici notre intérêt au second type de reproduction. La diversité apparaît aussi bien dans le comportement des partenaires sexuels que dans la morphologie de leur système reproducteur. Ce dernier comporte quatre éléments principaux : cellules germinales, gonades, conduits génitaux, organes copulateurs. Seul, le premier élément ne fait jamais défaut. Les trois autres n'existent que dans certains groupes zoologiques.

Dans trois groupes d'animaux didermiques (placozoaires, éponges et certains cnidaires), l'appareil reproducteur se réduit aux cellules germinales [1-3]. Celles-ci sont libres et se développent au sein de l'ectoderme ou de l'endoderme, ou encore dans l'espace compris entre ces deux feuillets. D'autres animaux possèdent des gonades, mais n'ont pas de conduits spécialisés pour en évacuer les produits. Une telle situation se rencontre chez une partie des cnidaires et beaucoup de tridermiques (bryozoaires, brachiopodes, vertébrés agnathes...) [3-5]. Plus nombreux sont les animaux dont le système reproducteur comporte trois éléments : cellules germinales, gonades et conduits génitaux. Enfin, quelques groupes pratiquent la fécondation interne et possèdent des organes copulateurs. C'est le cas pour la grande majorité des espèces terrestres.

Il est logique de penser que le système reproducteur s'est élaboré progressivement au cours de l'évolution. Nous supposons que les métazoaires les plus primitifs comportaient déjà deux catégories de cellules, germinales et somatiques [6]. Dès le début, les cellules germinales possédaient plusieurs propriétés distinctives, héritées de leurs ancêtres unicellulaires : l'immortalité, l'aptitude à réaliser la méiose, la capacité de fusionner avec un partenaire de sexe opposé, l'incapacité de former par elles-mêmes un tissu cohérent [6].

Les cellules germinales constituent l'élément le plus ancien du système reproducteur. Les autres éléments apparurent par la suite, à mesure que le soma se développait et se diversifiait. Ce schéma évolutif permet d'expliquer plusieurs propriétés du système reproducteur, qui ne se comprennent que si on les considère comme des vestiges du passé.

\section{Localisation des cellules germinales dans l'embryon}

Dans de nombreux groupes zoologiques, les cellules germinales apparaissent dès le début du développement embryonnaire [6]. On les appelle gonocytes aussi longtemps qu'elles ne manifestent aucun signe de différenciation mâle ou femelle. Les gonocytes se forment en dehors des ébauches génitales et les rejoignent par la suite. 
Quand ils se forment très précocement, comme c'est le cas pour les rotifères [7], les nématodes et les insectes [8], les gonocytes ne peuvent être assignés à aucun feuillet particulier [9]. Leur lieu d'apparition varie d'un type d'embryon à l'autre, mais se situe en surface ou en périphérie du germe, plutôt qu'en profondeur (figure 1). Cela reste vrai lorsque les cellules germinales deviennent détectables plus tardivement, comme chez les amphibiens anoures, les amphibiens urodèles et les mammifères, où les gonocytes s'individualisent respectivement dans l'endoderme [10], le mésoderme latéral [11] et le mésoderme extra-embryonnaire [12].

\section{A • Drosophila melanogaster (insecte diptère)}

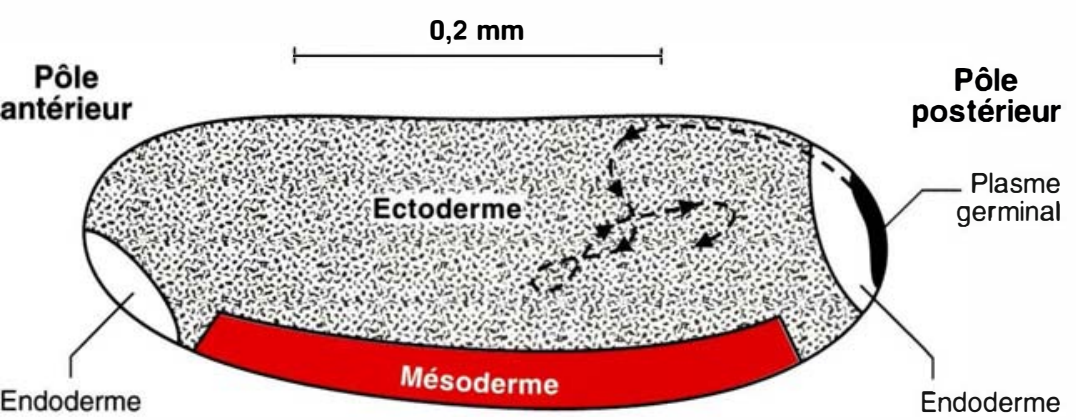

- Rana esculenta (amphibien anoure)

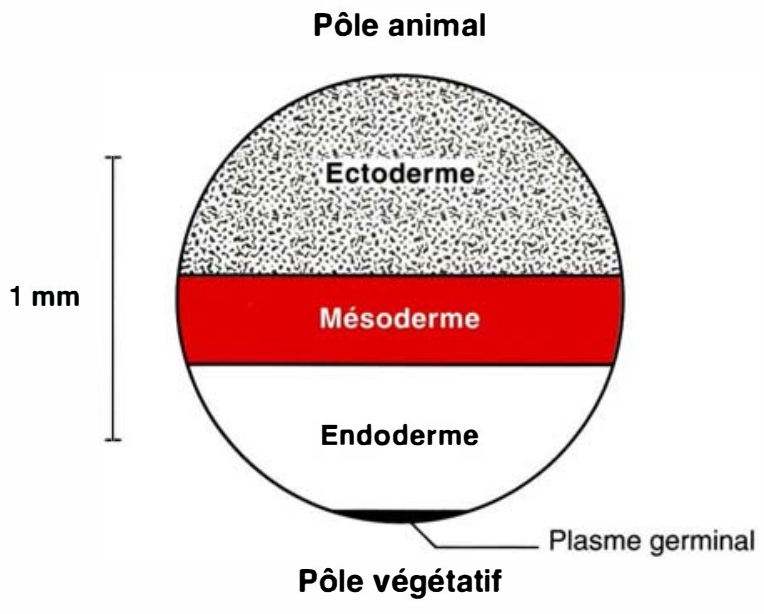

Figure 1. Position du plasme germinal dans un œuf d'insecte et d'amphibien. Les schémas présentés sont des plans d'ébauches, c'est-à-dire des projections sur la surface de l'œuf, des territoires occupés par les futures cellules ectodermiques, mésodermiques, endodermiques et germinales, après la division du cytoplasme initial. Cette partition ne s'accompagne d'aucun déplacement important de territoire. Par la suite, la gastrulation fera migrer le mésoderme et l'endoderme à l'intérieur de l'embryon. Plus tardivement, les gonocytes rejoindront les ébauches génitales, qui dérivent du mésoderme. La ligne sinueuse indique le trajet approximatif des gonocytes pendant le développement embryonnaire de la drosophile (A). II n'est pas possible de tracer l'itinéraire des gonocytes chez un amphibien (B), parce que la forme générale de I'embryon change considérablement pendant qu'il se développe. ISchéma A : d'après Campos-Ortega et Hartenstein [13], modifié. Schéma B : d'après Bounoure [10], modifié.)

Lorsque le germen s'individualise précocement, il est possible de délimiter un territoire ovulaire, dénommé plasme germinal, à partir duquel se formeront les gonocytes (figure 1). Ce territoire est censé contenir les déterminants germinaux [6]. Comme celle des gonocytes, la localisation du plasme germinal varie d'après le groupe zoologique. Nous supposons qu'un tel territoire existait chez les métazoaires les plus anciens, avant même que le soma se scindât en deux, puis trois feuillets embryonnaires. Sa position a ensuite fluctué dans les diverses lignées animales.

Pareille fluctuation n'est pas propre au plasme germinal. Elle a affecté la position de tous les territoires présomptifs de l'œuf. Il suffit, pour s'en convaincre, de comparer le plan d'ébauche d'un œuf d'amphibien (grenouille) avec celui d'un œuf d'échinidé (oursin). Dans les deux cas, la mise en place des trois feuillets embryonnaires se fait de manière comparable. Cependant, les deux types d'œufs diffèrent en ce qui concerne la disposition des territoires correspondant à chacun des trois feuillets. Dans l'œuf d'amphibien (figure 1), le futur mésoderme occupe une position équatoriale, entre l'hémisphère animal (pigmenté), à destinée ectodermique, et l'hémisphère végétatif, à destinée endodermique. Dans l'œuf d'oursin, c'est l'endoderme présomptif qui est intercalé entre le territoire ectodermique (animal) et le territoire mésodermique (végétatif) [14].

\section{Détermination et migration des gonocytes}

Pour les gonocytes, le premier événement important est la détermination, c'est-à-dire l'engagement dans la voie de différenciation germinale. Cet engagement est progressif. Pendant un certain temps, il reste réversible et les gonocytes demeurent totipotents. C'est ce qu'on observe chez les amphibiens : tant qu'ils sont libres, les gonocytes peuvent s'intégrer à divers tissus somatiques quand on les réimplante dans un embryon plus jeune [15]. Après leur inclusion dans les ébauches génitales, les gonocytes se déterminent de manière définitive, 
mais il est difficile de préciser quand cct événement se produit.

Avant même la migration des gonocytes, les cellules somatiques perdent apparemment l'aptitude à devenir germinales. Cette perte est plus ou moins précocc suivant que la lignéc germinale se constitue plus ou moins tôt dans l'cmbryon [6]. Après la colonisation des gonades, lc compartiment germinal et le compartiment somatique s'isolent complètcment. Cela interdit toutc métaplasie reproductivc, c'est-à-dire toute conversion d'une cellule somatique en cellule germinale. Unc telle transformation paraît exclue chcz les verté- brés. Privées de gonocytes, les gonades demeurent stériles $[16,17]$. Les gonocytes restent rarement à l'endroit où ils se sont formés. Chez les espèccs dépourvues de gonades, lcs cellules germinales se déplacent parmi les ccllules somatiques [2, 3] La mobilité des cellules germinales subsiste chez les animaux possédant des gonades. Elle se manifeste pendant le développement embryonnaire par la migration des gonocytes vers lcs ébauches génitalcs. Le mouvement cst cn général centripète. Il suit des chemins divers, puisque le lieu de formation des gonocytes varie suivant le type d'cmbryon (figure 1). Lc tra-

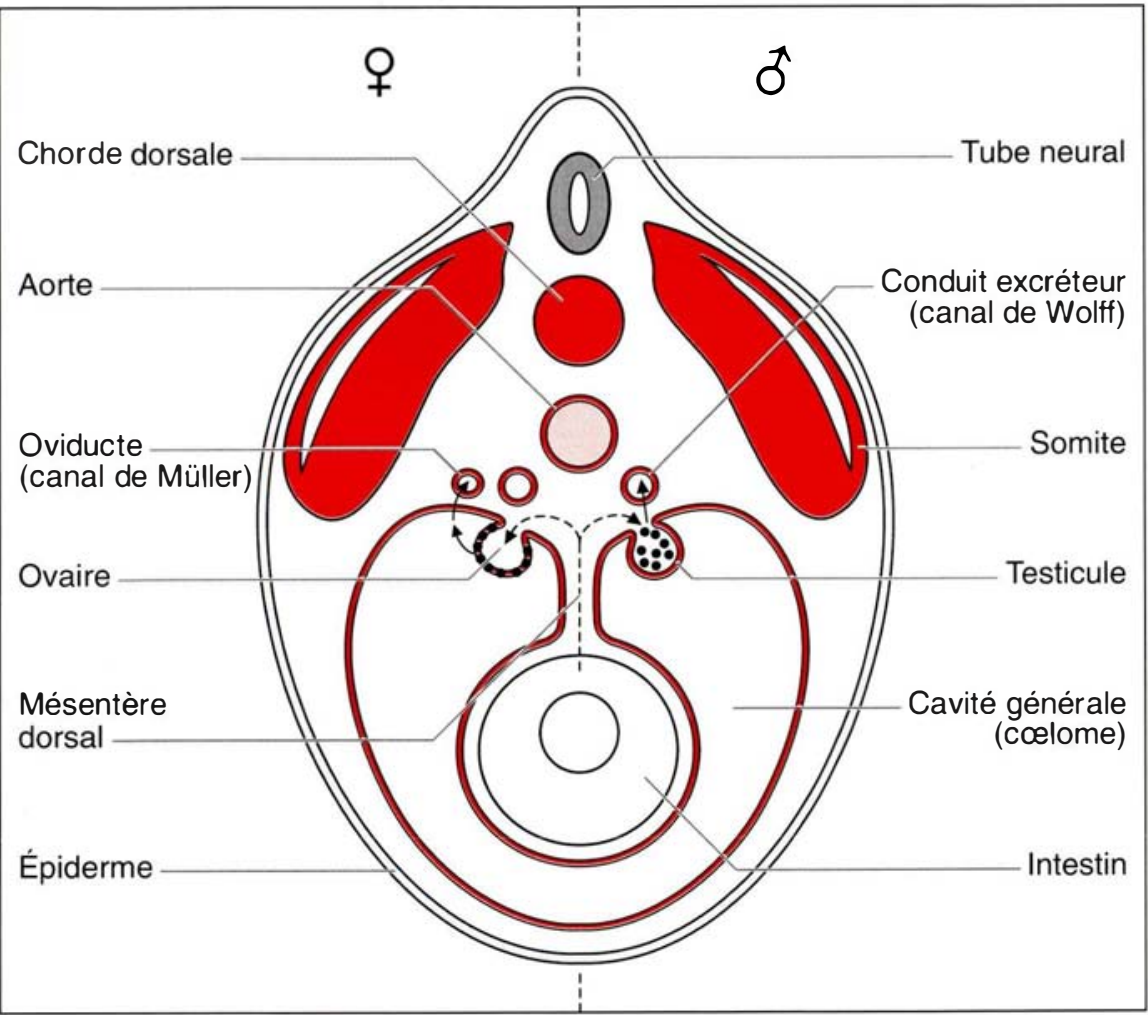

Figure 2. Migration des gonocytes dans un embryon de vertébré anamniote. Le schéma représente une coupe transversale dans la partie troncale de l'embryon, au niveau des ébauches génitales. Les tissus d'origine ectodermique sont en pointillé ; les tissus d'origine mésodermique sont en rouge ; le tube digestif, d'origine endodermique, est en blanc. Les lignes interrompues indiquent le trajet parcouru par les gonocytes (en noir) chez les amphibiens anoures. Les ébauches génitales se forment comme des replis de la paroi dorsale du cœlome. Elles comportent deux parties : une partie corticale et une partie médullaire. D'abord vides, les ébauches génitales sont envahies par les gonocytes. Si l'ébauche évolue en ovaire, c'est le cortex qui devient la partie principale de la glande. Si l'ébauche de gonade devient testicule, le cortex s'atrophie. La partie profonde de la glande, appelée médulla, se développe à partir de cellules mésodermiques qui ont envahi la cavité, et qui dérivent probablement du rein troncal (mésonéphros). Chez l'adulte, les ovules (à gauche) seront libérés dans la cavité générale, puis repris par les oviductes, qui relient cette cavité à l'orifice génital. Les spermatozoïdes (à droite) seront évacués jet des gonocytes a été bien étudié chez les vertébrés, et plus particulièrement chcz les amphibicns, les oiseaux et les mammiferes [17]. Par cxcmple, les cellules germinales des anoures quittent l'endoderme avant la fin dc l'cmbryogenèse, poursuivent lcur marche en direction dorsale, puis pénètrent dans les ébauches de gonade (figure 2) [10].

Nous interprétons la mobilité précoce des gonocytes comme un héritage du passé lointain où les animaux n'avaient pas de gonades pour accueillir leurs cellules sexuelles. En cette matière, l'cnchaînement des événcments embryonnaires reflètc celui des événements ćvolutifs. Pendant l'cmbryogenèse, lcs gonocytcs passent par une phase libre avant de s'enfermer dans les gonades. Chez les métazoaires primitifs, les cellules gcrminales se développaient librcment au sein du soma. Dans une étape évolutive ultérieure, les cellules sexuelles se réfugic̀rent dans un organe spécialisé : ovaire ou tcsticule.

\section{Structure des gonades}

Les gonades ont une origine somatique. Leur structure varie suivant le groupe zoologique. Certains animaux didermiques amorcent une concentration des cellules sexuelles. Au lieu d'être éparses, comme celles des éponges, les cellules germinales sont réunics en des organes rudimentaires, appelés ampoules génitales ou gonophores, selon leur degré d'organisation [19]. De telles formations existent chez les cnidaires de la classe des hydrozoaires (hydres et hydroméduses). Elles dérivent d'un scul feuillet ou des deux feuillets embryonnaires [19]. D'autres animaux didermiques possèdent de véritables gonades, où les cellulcs germinales sont étroitement associées avec des cellules somatiques. C'est le cas, par exemple, pour les cténaires [20], et pour quelques cnidaires appartenant à la classe des anthozoaires (coraux) et à celle des scyphozoaires (méduses communes) [3].

Les gonades des animaux tridermiques proviennent du mésodcrme. La structurc même du feuillet intermédiaire impose une distinction cntre deux grandes catégories de tridermiques : les acœlomates et les cœlomatcs [4]. Chez les premiers, lc méso- 
derme n'est pas organisé en vésicules closes. Les gonades des acœlomates peuvent être incluscs dans le parenchyme qui remplit l'espacc entre le tube digestif et la paroi du corps. Un tel agencement se rencontre chez les turbellariés [21]. Il arrive aussi que les gonades flottent dans le liquide remplissant la cavité corporelle. Cette disposition se présente lorsque le mésoderme est réduit, comme chez les nématodes [22].

Les cœlomates réunissent la majeure partie des grands groupes zoologiques (annélides, mollusques, arthropodes, échinodermes, vertébrés...). Le mćsoderme se creuse cn une série de sacs dont l'ensemble forme le colome et peut confluer en un vaste espace intćrieur, appelé cavité générale, qui contient les viscères. Le creusement du mésoderme retentit sur la position des gonades. Celles-ci sont souvent rejetées à la périphérie du cœlomc. Ainsi, les ébauches génitales des vertébrés apparaissent comme des replis de la paroi dorsale du colome, suspendus dans la cavité générale (figure 2).

\section{Origine des gonades}

Par leur structure et leur mode de formation, les gonades des animaux didermiques diffèrent profondément de celles des animaux tridermiques. Il est donc vraisemblable que ces organes apparurent indépendamment dans ces deux groupes zoologiques. Dans l'une et l'autre lignéc animale, les gonades se mirent à recruter des éléments germinaux qui cxistaient depuis longtemps. Cette mobilisation se reproduit à chaque génération, lorsque les ébauches génitales se peuplent de gonocytes formés en dehors d'elles.

Peut-on dire que les gonades de tous les animaux tridermiques sont homologues? Autrement dit, les gonades des nématodes, des mollusques, des arthropodes, des vertébrés sont-elles le fruit d'un seul événement évolutif ou de plusieurs événements indépendants? Dans l'état actuel de nos connaissances, il est difficile de prendre parti pour l'une ou l'autre hypothèse. On serait plutôt tenté de choisir la seconde possibilité, étant donné la diversité que revêt le modc de formation des gonades.

Un argument en faveur de la pre$\mathrm{m} / \mathrm{s} \quad n^{\circ} \quad 6-7$ vol. 9, juin-juillet 93 mière possibilité pourrait être apporté par la biologie moléculairc. Peut-être existe-t-il des gènes homologues qui gouvernent la formation des gonades chez tous les tridermiques, attestant leur origine commune. La découverte de tels gènes semblc improbable. Toutefois, l'avenir peut réserver des surprises. Jusqu'à récemment, personne n'imaginait que chez des animaux aussi différents que les insectes et les vertébrés, des batteries de gènes homologues gouvernent le modelage de l'embryon. C'est pourtant ce que l'on observe: les chromosomes des deux groupes contiennent des gènes disposés dans le même ordre que celui où ils s'expriment suivant l'axc antéro-postérieur de l'animal [23].

\section{Fonctions des gonades}

Les gonades des vcrtébrés influencent les gonocytes avant même d'être colonisćes par eux (figure 3, p. 756). Elles émettent une substance qui attire les cellules germinales $[24,25]$. La molécule attractive pourrait appartenir à la famille des facteurs de croissance [25]. L'effet chimiotrope joue un rôle essentiel dans la migration des gonocytes [25]. Cet cffet n'est peutêtre pas limité aux vertébrés. Si tel était le cas, il faudrait considérer le pouvoir attractif des gonades comme une acquisition évolutive très ancienne, remontant à l'origine même des glandes génitales. Il est possible que ce soit l'apparition précoce du chimiotropisme qui permit la variation observée dans le lieu de formation des gonocytes. Il importe peu que ceux-ci suivent des chemins divers pour gagner les ébauches de gonade. Attirés par leur cible, ils finissent par l'attcindre, quels que soient leur point de départ et leur itinéraire.

Dans plusieurs groupes animaux, la fonction endocrine des gonades n'est pas restreinte à la vie embryonnaire. Les gonades adultes des vertébrés produisent les hormones mâles et femelles, responsables de l'apparition des caractères sexuels secondaires. Les deux types d'hormones sont des stéroïdes dérivés du cholestérol. Des hormones de même nature ont été mises en évidence chez les échinodermes [26]. En dehors des vertébrés, il y a peu de groupes zoologiques où l'existence d'hormoncs sexuelles a pu être démontréc sans ambiguïté. Les crustacćs possèdent de telles hormones. Certains de ces animaux (les malacostracés) ont une paire de glandes androgènes, accolées au tractus génital mâle [27]. Implantée dans unc femelle, la glande androgène transforme les ovaires en testicules [27]. La nature de l'hormone androgène n'a pas été identifiée.

Les gonades n'agissent pas seulement à distance. Elles jouent un rôle actif dans la gamétogenèse. En premier lieu, elles procurent une protection mécanique aux cellules germinales. Dans beaucoup d'espèces, l'ovaire enveloppe les oocytes d'un follicule complet, qui sécrète une coque plus ou moins rćsistante, destinée à protéger l'œuf après la ponte. Les gonades ont aussi une fonction inductive et trophique, puisqu'elles produisent ou font transiter les substances nécessaires à la différenciation des cellules germinales. On peut également attribuer aux gonades une fonction coordinatrice. En rassemblant en un même lieu les cellules germinales, il scmble plus facile de déclencher do façon concertée leur différenciation, puis leur émission.

A l'exception des étapes initiales (individualisation et migration), c'cst à l'intérieur des gonades que s'accomplisscnt les phases principalcs de la gamétogenèse (figure 3 ). Toutes ces phascs devaicnt exister chez les animaux primitifs, avant l'apparition des glandes sexuelles et la capture des cellules germinales. Parmi les étapes évoquées, certaines sont passées sous le contrôle des gonades; d'autres ont conservé une autonomie totale ou partielle (figure 3). Par la force des choses, une telle autonomie persiste chez les animaux dépourvus de gonades.

\section{Sexualisation des gonocytes}

Après leur inclusion dans les gonades, les gonocytes se différencient en oogonies et en spermatogonies, qui sont reconnaissables par leur morphologie (figure 3). La sexualisation des gonocytes est sous la dépendance du soma. Elle ne dépend pas de la constitution génétique des cellules germi- 


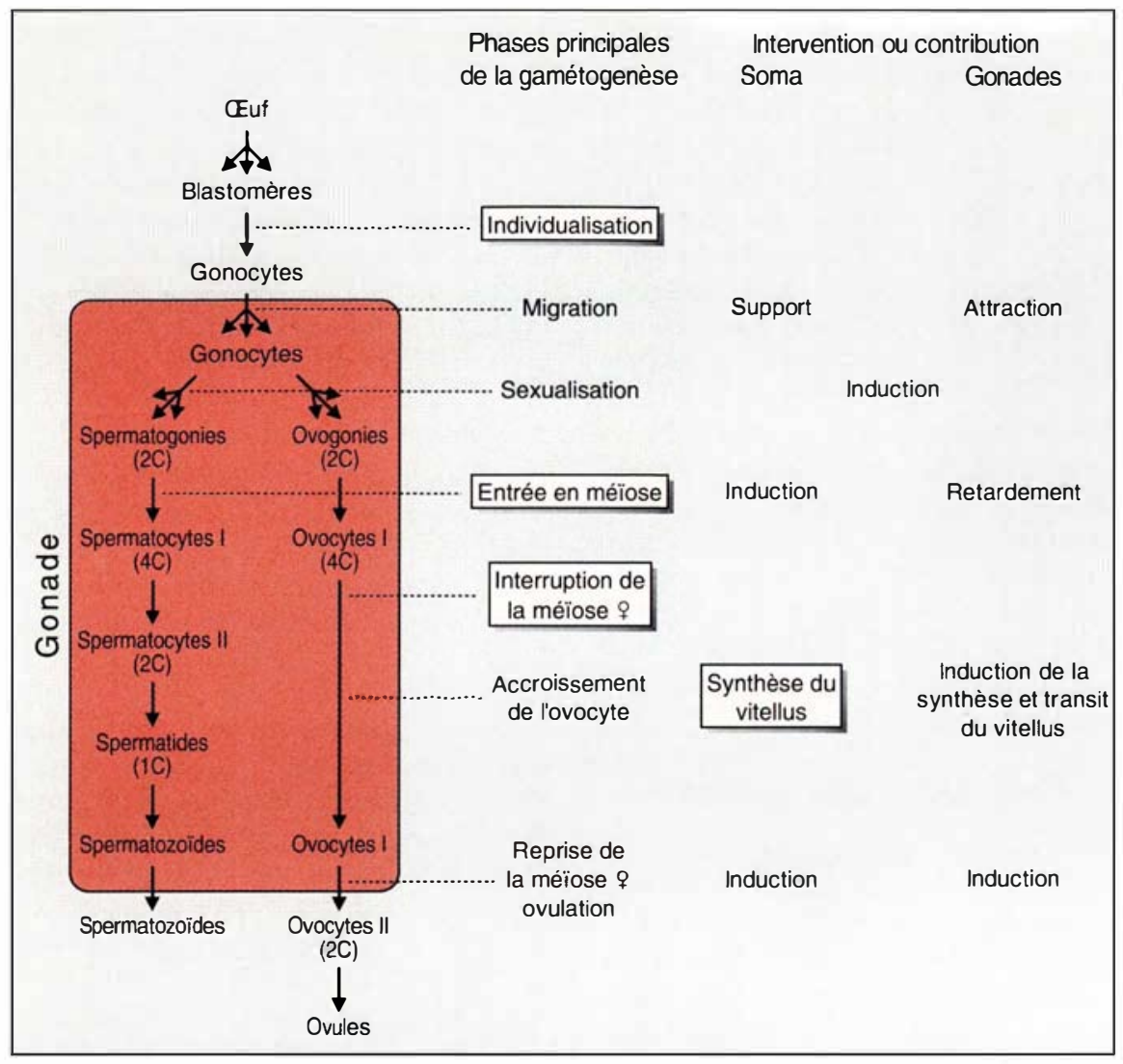

Figure 3. Différenciation des cellules germinales. Le schéma présenté concerne surtout la gamétogenèse des vertébrés. II montre les principales phases de la vie des cellules germinales avant, pendant et après leur inclusion dans la gonade. Les étapes de multiplication sont indiquées par des flèches triples. Les phases postérieures à l'entrée en méiose se rapportent uniquement aux cellules femelles. Les cadres mettent en évidence les processus qui jouissent d'une autonomie totale ou partielle par rapport à la gonade, en ce sens que celle-ci ne joue pas le rôle principal dans leur déroulement. Au moins deux incertitudes subsistent. Au sujet de la détermination du sexe, il est difficile de préciser la contribution respective du soma et des gonades. Une autre incertitude a trait à l'entrée en méiose. L'intervention des gonades semble peu importante. Chez les mammifères, elle pourrait se limiter à une influence retardatrice du testicule.

nales. Celles-ci peuvent donc subir une différenciation non conforme à lcur génotype [17].

Chez la drosophile, l'influence somatique n'est pas entièrement déterminante pour spécifier le sexe des cellules germinales. Elle se conjugue à des facteurs génétiques, si bien qu'une cellule de génotype mâle ou femelle ne donnera pas de gamète fonctionnel de sexe opposć si elle se différencie dans un soma non conforme [28]. Des observations semblables ont été faites chez les mammifères. Par excmple, une cellulc germinale femelle $(\mathrm{XX})$ se transforme en un environnement testiculaire [29]. Toutefois, la cellule dégénère avant le début de la mćiose [29]. Il n'en va pas de même pour lcs amphibiens et les télćostéens [17]. Après transplantation de gonocytes dans des cmbryons de sexe opposé, on obtient des mâles qui produisent des spermatozoïdes dérivant de cellules génétiquement femelles [17, 30]. L'općration crée aussi des femelles qui pondent des ovules provenant de gonocytes génétiquement mâles [17, 30]. On arrive à des résultats similaires en changeant le sexe somatique par l'administration d'hormones sexuelles [31].
Il est logique de penser que ce sont les gonades elles-mêmes qui aiguillent les gonocytes dans la voic mâle ou femelle. Unc observation faite chez lcs urodèles soutient cette opinion. $\mathrm{Si}$ l'on greffe, sur un embryon, du mésoderme latéral provenant d'un embryon de l'autre sexe, une gonade et des gamètes se forment à partir du transplant. Le sexe des gamètes correspond à celui de la gonade, et non à celui du soma qui l'entoure [32]. En matic̀re de détermination du sexe, on peut se demander si la domination du soma cxistait déjà chcz les mćtazoaires archaïques, qui ne possédaient pas de gonades. S'il en ćtait ainsi, les glandes génitales n'auraient fait que reprendre à leur compte une propriétć partagće à l'origine par l'ensemble des cellules somatiques. Un ćlćment de réponse est fourni par l'étude des hydraires, et plus particulic̀rement de l'hydre d'eau douce, qui nc possc̀de pas à proprement parler de gonades [19]. Des expériences de greffe ont conduit à conclure qu'une cellule germinale femelle subit la spermatogenc̀se lorsqu'elle est placéc dans une hydre mâlc [19]. Mais cettc interprétation est contestćc.

On pourrait croire que ce sont les hormones sexuelles produites par les gonades qui orientent la différenciation des ccllules germinales. Tel n'est pas le cas cependant, tout au moins pour les vertébrćs. Dans un foctus mâle de souris, une cellule germinale de génotype mâle (XY) évolucra en ovocyte, si clle ne rejoint pas l'ébauche testiculaire et s'ćgare dans la glande surrénale [33]. La différenciation femelle est donc une proprićtć intrinsèque des cellules germinales, que sculc pourra modifier l'inclusion dans un testicule, et non pas seulement l'exposition à l'hormone mâle circulante. Une conclusion similaire émerge des études faites sur les crustacés : cn l'absence de glande androgène, les gonocytes subissent l'ovogenèse, même si leur constitution génétique est mâle [27]. Selon toutc vraiscmblance, l'hormone androgène agit de manière indirecte sur la différenciation en spermatozoïdes des cellules germinales, en transformant l'ovaire en testicule.

Il paraît étonnant que les cellules germinales de souris puissent subir un début d'ovogenèse en dehors des 
ovaires [33]. Nous attribuons une signification évolutive à la différenciation ectopiquc des gonocytes. Ce processus subsisterait comme le vestige d'un mode de formation archaïque des cellules sexuelles, antćrieur à l'apparition des ovaires.

\section{Entrée en méiose}

Après une phasc de multiplication, les gonics entrent en méiose (figure 3). Chez aucun animal, les signaux d'entrée en méiose n'ont été identifiés. Dans beaucoup de groupes zoologiques, la méiose se déclenche de façon périodique et souvent saisonnière. Il est donc probable quc les signaux proviennent, directement ou indircctement, du système nerveux. Si c'était le cas, il faudrait conclure que la gonade ne joue qu'un rôle limité dans le déclenchement de la méiose. Cette situation cst peut-ĉtre primitivc, analogue à celle qui prévalait chez les métazoaires archaïques.

Chez des animaux très différents (les mammifères et les nématodes), on a pu constater que la gonade exerce une influence inhibitrice sur l'entrée en mćiose des ccllules germinales. Celle-ci se ferait de manière autonome ou serait déclenchée par des facteurs externes. Le testiculc de la souris retarde jusqu'après la naissance l'entrée en méiose des spermatogonies [29]. Cette action ne s'excrce qu'à courte distance, puisque la glande surrénalc des fotus mâles peut contenir des ovocytes en mćiose [33]. On ignore la nature de l'inhibiteur produit par le testicule. De son côté, la méiose femelle commencerait dc façon autonome au cours de la vie fœitale [29]. C'est ce que suggère l'étude des cellules germinales égarées dans la glande surrénalc: clles entrent en méiose cn même temps que celles qui sont incluses dans les ovaires [29, 33]. Une autre opinion attribue le déclenchement de la méiose dans l'ovaire à une hypothétique substance inductrice, qui serait produite par le rein fotal [34].

L'ovotestis du nématode Caenorhabditis inhibe l'entréc en mćiose des cellules germinales qu'il contient [35]. L'inhibiteur émane d'une seule cellule, située au pôle postćrieur de l'organe. Les cellules germinales situées à proximité prolifčrent. Elles n'entrent en méiose que lorsqu'elles sont suffisamment éloignécs de la pointe de l'ovotestis. Si l'on détruit la ccllule régulatrice, toutes les cellules germinales subissent la méiose, sans se diviser au prćalable. On a pu identifier un gène ( $g l p-1)$, dont l'inactivation empêche lcs cellules germinales de rćpondre à l'inhibiteur produit par la gonade [35].

\section{Interruption}

\section{et reprise de la méiose}

La mćiose mâlc se déroule sans interruption. Il n'en va pas dc même pour la méiose femclle, qui s'arrête en prophase de la première division. L'ovocyte profitc de cette pause pour s'accroître (figure 3). La méiose ne reprend que lorsque l'ovocyte a atteint sa taille maximalc. Chez la plupart des animaux, l'ovulation a lieu pendant ou après l'achèvement de la méiose. Ainsi s'achèvc la phase captive de la vic des cellules germinales femelles.

On nc connaît pas le mécanisme commandant l'arrêt de la méiose. L'ovocyte semble déclencher cet événement de façon autonome, sans que l'ovaire intervienne. C'est démontré chez la souris : les cellules germinales d'un fœtus mâle cntament, puis interrompent la méiose quand clles s'égarent dans la glandc surrénale [33]. En cctte matière, l'autonomic de l'ovocyte est probablcment une acquisition évolutive très ancienne, qui aurait précédé l'apparition des gonades. En tout cas, elle existe chez les animaux actuels qui ne possèdent pas d'ovaires [2].

Dans plusieurs groupcs zoologiques, on a identifié les signaux ordonnant la reprise de la méiose femelle. Chez les astérides (ćtoiles de mer) et les vcrtćbrés, ces signaux proviennent du système nervcux. Ils induisent une réponse dans les cellules des follicules ovariens, qui sécrètent une hormone de maturation. L'hormone des vertébrćs est un stéroïde : la progestérone. Celle des astérides est une base purique : la 1-méthyladénine [8]. Les hormones de maturation font apparaître dans l'ovocyte un facteur particulier : le MPF (maturation promoting factor) [6]. Celui-ci déclenche à son tour la reprise de la méiose, dont la première manifestation est la rupture de l'enveloppe nucléaire $[6,17]$.

\section{Accroissement des ovocytes}

Pendant l'interruption de la méiose, l'ovocyte grossit considérablement (figure 3). Chez les espèces ovipares, sa taille devient souvent gigantesque. Pour s'accroîtrc, l'ovocyte reçoit du soma toutcs les substances nutritives dont il a besoin. La plus grande partie de ces substances se trouve dans lc vitellus. Celui-ci contient surtout des lipides et des protéines. Ces dernières constituent un stock d'acides aminés, que l'embryon utilisera pour assembler les protéines indispensables à son développement.

Ce sont des ccllules extérieures à l'ovaire qui synthétisent une partie ou la totalité des protéines vitellines. L'originc de ces protćincs varie d'un groupe zoologique à l'autre. Chez les nématodes et les vertébrés, elles sont produites par des organes de nature endodermique. Il s'agit de l'intestin pour les premiers et du foie pour les seconds [36]. Le vitcllus des échinides a une provenance mixtc : intestin et ovaire [26]. Cclui des insectes et des crustacés est synthétisé dans une formation mésodermique : le corps gras ou lc tissu adipeux [37, 38].

Chcz tous les animaux étudićs, à l'exception des diptèrcs supérieurs (drosophile, moustique), les composants protéiques du vitellus sont synthétisés, puis sécrétés sous la forme d'un précurscur commun, appelé vitellogénine [36]. Le précurseur est vćhiculé par les fluides corporels. Il pénètre enfin dans les ovocytes pour y être converti en produits insolubles. Chez les vertébrés, où l'ovocyte cst entouré d'un follicule, la vitellogénine passe par les interstices subsistant entre les cellules folliculaires, sans transitcr par cellesci [39]. L'ovaire intervient quand même dans la vitellogenèse, puisque la production de vitellogénine est déclenchće par une hormone d'origine ovarienne : l'œstradiol [36].

Il scmble étonnant qu'cn matière trophique, les ovaires ne jouent pas le rôle le plus important, puisque les ovocytes sont en contact étroit avec les cellules ovariennes. Il existe une explication évolutive pour la formation exogène des protéines du vitellus. Ce serait un mode très ancien de 
nutrition des ovocytes, qui existait avant même l'apparition des gonades. C'est ce que suggère l'étude de la vitellogénine dans différentes espèces.

La vitellogénine des vertébrés est homologue de celles des nématodes, des insectes (à l'exception des diptères supérieurs), et peut-être aussi des échinides [26, 36, 37, 40]. La vitellogénine est donc une protéine très ancienne, que possédait déjà l'ancêtre commun des acœlomates et des cœlomates [36, 40]. Il est probable que la vitellogénine fut inventée par un métazoaire plus primitif encore, qui n'avait ni mésoderme ni gonades. Nous supposons que chez cet animal, la vitellogénine était sécrétée dans le milieu intérieur par les cellules de l'endoderme. Elle était ensuite captée par les ovocytes. Les animaux tridermiques cnfermèrent les cellules germinales dans un repli du mésoderme, mais conservèrent deux acquis de leurs prédécesseurs, que l'on retrouve dans plusieurs groupes zoologiques actuels : synthèse de la vitellogénine par le feuillet interne ; passage direct dc la protéine du milicu intérieur aux ovocytes, sans transit par les cellules de l'ovairc.

\section{Émission des gamètes}

Les gonades des acœlomates sont reliées à la paroi du corps par des conduits de faible longueur, dérivés du mésoderme. Chez les cœlomates, le creusement du mésoderme a des conséquences considérables sur l'émission des cellules sexuelles. La paroi de la gonade se rompt, relâchant les gamètes dans le cœlome ou la cavité générale. Un tel phénomène se produit dans de nombreux phylums (embranchements) d'invertébrés, à l'exclusion notable des arthropodes, dont le cœlome est très réduit, et des échinodermes [4].

Les choses se passent de la même manière pour beaucoup de vertébrés. Le meilleur exemple est celui des agnathes: les produits sexuels sont libérés par les gonades dans la cavité générale, puis émis par un ou deux pores abdominaux [5]. La situation est plus complexe chez les gnathostomes. Dans la plupart des groupes (chondrichthyens, amphibiens, reptiles, oiseaux et mammifères), seules par la cavité générale [18]. Les ovules sont évacués par des conduits spécialisés, que n'ont pas les agnathes [5].

Pour beaucoup de cœlomates, la gonade offre donc une ouverture temporaire dans la paroi du cœlome, par où les cellules germinales font irruption dans la cavité générale (figure 2). Parties d'une région périphérique de l'embryon, les cellules germinales finissent par atteindre, au terme de leur différenciation, la partie la plus interne de l'organisme. Cette marche centripète est interrompue par un séjour prolongé dans la gonade.

Comment interpréter le transit des cellules sexuelles par le cœlome? Il pourrait s'agir d'une acquisition unique de l'ancêtre commun des cœelomates actuels, conservée par la majeure partie de ses descendants. Si cette hypothèse est correcte, il devrait exister chez divers cœlomates, des gènes homologues, dont la fonction est de provoquer la rupture de la paroi de la gonade, donc la libération des gamètes dans le cœlome ou la cavité générale.

Pour les animaux primitifs, il y avait peut-être un avantage fonctionnel à faire transiter les gamètes par le cœlome: le liquide cœlomique est riche en nutriments et bien protégé contre les variations du milieu. Il est possible que les cellules germinales des métazoaires archaïques accomplissaient une partie de leur différenciation à l'intérieur du cœlome. Une telle situation se rencontre chez certains invertébrés actuels : échiuriens, sipunculiens, annélides oligochètes [41-43]. A un stade évolutif ultérieur, le séjour dans la gonade se serait prolongé, jusqu'à couvrir la quasi-totalité de la gamétogenèse.

Si le transit colomique a pu s'instaurer chez les animaux primitifs, c'est sans doute parce que le cœlome communiquait déjà avec l'extérieur. Les néphridies assurent une telle communication. Typiquement, une néphridie s'ouvre dans le cœlome par un orifice appelé néphrostome. Son extrémité distale débouche à l'extérieur ou dans l'intestin. Des cellules ciliées créent un courant liquide qui entraîne les déchets accumulés dans la cavité cœlomique.

Les néphridies sont utilisées par de nombreux cœlomates (sipunculiens, échiuriens, phoronidiens, brachiopodes, annélides, mollusques primitifs...) pour l'émission des cellules sexuelles [4]. Dans ce cas, le conduit excréteur possède une double fonction. Il arrive aussi que les ovules et les spermatozoïdes soient émis par des conduits particuliers, dérivés du mésoderme : les gonoductes [4]. Le cœlome peut alors être relié à l'extérieur par deux types de canaux, les uns servant à l'évacuation de l'urine, les autres à celle des cellules reproductrices. Néphridies et gonoductes coexistent chez certains annélides [44].

\section{Les conduits génitaux des vertébrés femelles}

Chez tous les vertébrés à l'exclusion des agnathes, le système excréteur est étroitement associé à l'évacuation des gamètes. Les connexions urogénitales varient suivant le sexe et le groupe zoologique. Les études d'anatomie comparée permettent de supposer que ces connexions n'existaient pas chez l'ancêtre des vertébrés actuels (figure 4). Les cellules sexuelles étaient relâchées par les gonades dans la cavité générale. La fonction excrétrice était assurée par un rein métamérique, dérivé du mésoderme, se formant comme un diverticule de la paroi du cœlome [45]. Le rein archaïque comportait un néphrostome par métamère, relié à un conduit commun, appelé uretère primaire, qui débouchait dans le cloaque (figure 4). L'émission des cellules sexuelles se faisait soit par le rein [45], soit par des pores abdominaux, comme cela se produit chez les agnathes actuels [5].

Une organisation que l'on suppose ancestrale subsiste dans le rein embryonnaire des agnathes, de certains ostéichthyens (poissons osseux) et amphibiens : les néphrons sont disposés de façon métamérique et s'ouvrent dans le cœlome par un néphrostome [45]. Ce rein, appelé pronéphros, est localisé au niveau du péricarde, dans la partie antérieure du corps. Au cours de l'évolution, les néphrons les plus antérieurs perdirent leur fonction urinaire. Celle-ci fut reprise par des reins plus postérieurs, apparaissant plus tardivement au cours de l'embryogenèse. Les anam- 
niotes adultes et les embryons des amniotes développent un rcin troncal (mésonćphros), dont lc conduit collecteur porte le nom de canal de Wolff (figure 4). Le mésonéphros des amniotes adultes perd à son tour toute fonction excrétrice. Il est remplacé par un rein postérieur (métanéphros), qui possède ses propres canaux évacuateurs, appelés uretères secondaires.
Bien qu'ayant perdu sa fonction urinaire, le pronéphros a pu subsister comme un diverticule s'ouvrant dans le colome. Certains auteurs en font dériver le pavillon (ostium) de l'oviductc $[9,45]$. Le pavillon se scrait formé à partir d'un ou de plusieurs néphrostomes fusionnés (figure 4). Une telle filiation s'observe très bien dans l'embryon de sélacien, moins facilement dans celui d'amphibien.

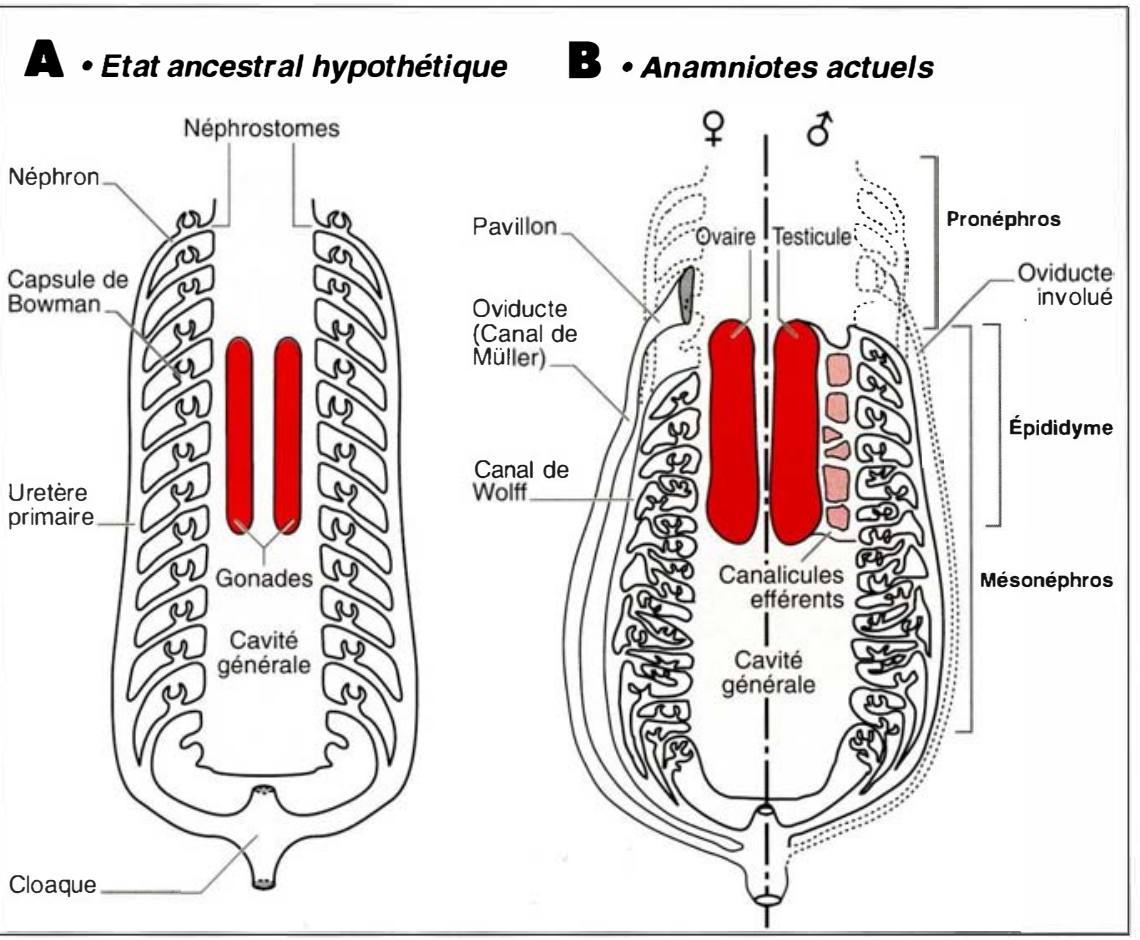

Figure 4. Connexions urogénitales chez les vertébrés. Chez les vertébrés primitifs (A), aucune connexion ne devait exister entre les gonades et le système excréteur. Les cellules sexuelles étaient relâchées dans la cavité générale. Les reins comportaient une série métamérique de néphrons débouchant dans un canal collecteur raccordé au cloaque : I'uretère primaire. Un néphron primitif comprend un entonnoir cilié s'ouvrant dans le cclome (le néphrostome) et un tubule, dont la paroi se dilate en une chambre à paroi mince lla capsule de Bowman), déprimée en cupule sous la poussée d'un peloton de vaisseaux sanguins (le glomérule). On trouve une telle organisation dans le pronéphros, rein antérieur qui fonctionne dans l'embryon de certains vertébrés inférieurs. L'urine élaborée par le pronéphros est évacuée par l'uretère primaire. Chez les vertébrés adultes (B), le pronéphros n'est plus fonctionnel. Les anamniotes le remplacent par un rein troncal (mésonéphros), dont les néphrons se branchent sur l'uretère primaire, appelé canal de Wolff en tant que conduit évacuateur du mésonéphros. Devenu inopérant, le pronéphros ne disparaît pas complètement. Un entonnoir se constitue à partir d'un néphrostome lou d'un groupe de néphrostomes) antérieur. Ce sera le pavillon de l'oviducte. Le pavillon se raccorde au cloaque par un nouveau conduit : le canal de Müller. L'embryon mâle forme un oviducte, qui involue rapidement dans la plupart des groupes. Le testicule établit une connexion avec le mésonéphros. Chez les amphibiens et les poissons (excepté les téléostéens), la liaison se fait avec la partie antérieure du mésonéphros, qui devient l'épididyme. Les spermatozoïdes passent donc à travers le rein pour rejoindre le canal de Wolff. (D'après Goodrich [45], modifié.)

Mais chez la plupart des autres vertébrés, le pavillon apparaît sans aucune trace visible de relais pronéphrotique [45]. Pour former un oviducte fonctionnel, il fallait encore relier l'ostium au cloaque. Un nouveau conduit apparut : le canal de Müller (figure 4). L'origine de ce dernier est difficile à établir car il se constitue apparemment par des mécanismes différents dans les divers groupes de vertébrés [9, 45, 46].

Suivant les groupes, le canal de Müller se différencie de manière variable. Chez les vertébrés ovipares et certains vivipares, l'oviducte acquicrt une fonction glandulaire, sécrétant les enveloppes qui entoureront l'œuf après la ponte, ou les substances nutritives (le lait utérin) destinées à l'embryon. Chez d'autres vertébrés vivipares (sélaciens, mammifères), lc canal de Müller se différencie en utérus, où l'embryon est nourri par un placenta. Il est clair que ces spécialisations n'auraient pas été possibles si le canal de Müller avait une fonction cxcrétrice, comme le canal de Wolff. L'évacuation de l'urine semble incompatible avec la sécrétion des envcloppes ovulaires, ainsi qu'avec la viviparité. Dans les deux ćventualités, l'œuf ou le fœtus obstruerait de façon prolongée le conduit excréteur.

\section{Les conduits génitaux des vertébrés mâles}

La petite taille des spermatozoïdes n'empêche pas leur émission par le même conduit que l'urine. Chez tous les vertébrés à l'exception des agnathes, les cellules sexuelles mâles ne transitent plus par la cavité générale. Le testicule des poissons (hormis les téléostéens) et des amphibiens ćtablit une connexion directe avec le mésonéphros. Les canalicules efférents du testicule se raccordent à des néphrostomes. Cela permet le passage des spermatozoïdes vers le canal de Wolff, qui devient donc un conduit mixte, évacuant à la fois l'urine et les spermatozoïdes (figure 4). On appelle épididyme la partie du rein utilisée pour le transit des cellules sexuelles mâles. Chez les amniotes, l'épididyme est le seul vestige fonctionnel du mésonéphros. Le canal de Wolff se transforme en canal déférent [9]. Pour les vertébrćs mâles, une consé- 
quence importante découlc de l'établisscment d'unc connexion urogénitale : à la fin de leur différenciation, les ccllules germinales empruntent pour quitter le testiculc lc même chemin que cclui qu'elles ont emprunté pour pénćtrer dans l'ébauche génitale (figure 2). En quelque sortc, clles rcbroussent chemin, contraircment aux cellules germinales femclles, qui poursuivent leur marche centripc̀te en tombant dans la cavité génćrale (figure 2). Du point de vuc cmbryologique, la connexion entre le testicule ct le rcin cst assez facile à comprendre si l'on admet, comme lc font plusicurs auteurs [9, 47], que lc testiculc dérive en partic du mésonéphros. Envahissant la partie crcusc de l'ćbauche génitale, les ccllules mésonćphrotiques fourniraient à la fois la médulla de la gonade (figure 2), ct les canaliculcs efférents qui relient les tubes sćminifères au rcin (figure 4).

La plupart des gnathostomes mâles sont dépourvus d'oviductes. Toutcfois, leur cmbryon ćdifie des canaux dc Müller, qui nc tardent pas à involucr (figure 4), sous l'action d'une substance produite par les cellules de Scrtoli du testicule : l'hormonc antimüllćriennc [46]. Cellc-ci est homologuc du facteur de croissance TGF$\beta[48,49]$, mais son mode d'action reste à ćlucider.

\section{Conclusion}

On pcut trouver abcrrante ct antiéconomique l'ćdification du système reproductcur, puisqu'il arrive fréquemment que l'embryon modific ou même supprime cc qu'il vient de construire. En cette matière comme cn bcaucoup d'autrcs, l'œuvre de la nature s'apparente au travail du bricolcur plutôt qu'à cclui de l'ingénicur. On y voit plus d'opportunisme que de rationalité [50]. L'étude des phénomèncs évolutifs montre que toutc structure nouvclle s'ćlabore à partir d'unc structure précxistante. Mais il arrive que l'élément ancien subsistc et fasse unc apparition fugitive dans l'cmbryon. Cette tendance naturclle cst instructive pour le biologistc : il trouvera dans le dévcloppement cmbryonnaire la trace d'événcments ćvolutifs même très reculés. Ces événcments sont conservés dans la mémoirc des espèces sous la forme de gènes agissant de manière transitoire sur le déroulement de l'embryogenèse.

Certains traits archaïques subsistent dans la genèse et le fonctionnement de l'appareil urogénital. Par exemple, la plupart des vertébrés maintiennent une discontinuité entre l'ovaire et son conduit évacuateur, si bien que l'ovule doit accomplir un court trajet dans le cœlome avant de rejoindre l'oviducte (figures 2 et 4). En fait, le transit par le cœlome est un phénomène très répandu dans le monde animal. Il est donc d'origine très ancienne. Il a été conservé par les gnathostomes femelles, mais perdu par les mâles, qui utilisent les conduits urinaires pour l'évacuation des spermatozoïdes (figures 2 et 4 ).

Un trait plus archaïque encore concerne l'origine des gonocytes. Il semblerait normal que ceux-ci apparaissent au sein même des gonades. Chez aucun métazoaire, les choses ne se passent de cette façon. Partout, les gonocytes se forment en dehors des ćbauches génitales. Une telle bizarreric ne peut avoir qu'une explication évolutive. Nous supposons que les gonocytes existaient bien avant que les gonades ne fussent inventées par les animaux tridermiques [6]. L'apparition des glandes génitales ne modifia ni le lieu ni le mode de formation des gonocytes. Simplement, elle transforma l'errance des cellules germinales en migration ordonnée

\section{H. Denis}

Professeur à l'université Pierre-et-MarieCurie. Centre de génétique moléculaire Cnrs, avenue de la Terrasse, 91198 Gifsur-Yvette Cedex, France.

\section{J.-C. Lacroix}

Professeur à l'université Pierre-et-MarieCurie. Laboratoire de génétique du développement, 7, quai Saint-Bernard, 75230 Paris Cedex 05, France.

\section{Remerciements}

Les auteurs remercient Paul Berreur, Alain Collenot, Hervé Le Guyader, André Mazabraud, Linda Sperling et Maurice Wegnez pour leurs critiques et leurs conseils pendant la préparation du manuscrit de cet article.

\section{RÉFÉRENCES}

1. Grell KG, Ruthmann A. Placozoa. In Microscopic anatomy of Invertebrates, vol 2 : Placozoa, Porifera, Cnidaria and Ctenophora. New York : Wiley-Liss, 1991 : 13-27.

2. Tuzet O. L’origine de la lignée germinale et la gamétogenèse chez les spongiaires. In : Wolff $\mathrm{E}$, ed. L'Origine de la lignée germinale. Paris : Hermann, 1964: 77-111.

3. Tardent $\mathbf{P}$. The differentiation of germ cells in Cnidaria. In : Halvorson HO, Monroy $\mathrm{A}$, eds. The Origin and Evolution of Sex. New York: Liss, 1986: 163-97.

4. Grassé PP, Poisson RA, Tuzet O. Zoo logie. Tome I. Invertébrés. Paris : Masson, 1961.

5. Fontaine M. Classe des cyclostomes. Formes actuelles. In : Grassé PP, ed. Traité de zoologie. Tome XIII. Premier fascicule. Paris : Masson, 1958 : 13-106.

6. Denis H, Lacroix JC. L'origine de la lignée germinale et de la mortalité cellulaire. médecine/sciences $1992 ; 8$ : 695-702.

7. De Bcauchamp P. Classe des rotifères In : Grassé PP, ed. Traité de zoologie Tome IV. Fascicule III. Paris : Masson, 1965 : 1225-379

8. Davidson EH. Gene Activity in Early Deve lopment. Orlando: Academic Press, 1986.

9. Balinsky BI. An Introduction to Embryology Philadelphic : Saunders, 1975.

10. Bounoure L. La lignée germinale chez les batraciens anoures. In : Wolff E, ed L'Origine de la lignée germinale. Paris: Hermann, 1964: 205-34.

11. Nieuwkoop PD. Le problème de la lignée germinale chez les urodèles. In Wolff $\mathrm{E}$, ed. L'Origine de la lignée germinale. Paris: Hermann, 1964: 193-204.

12. Ginsburg M, Snow MHL, McLaren A. Primordial germ cells of the mouse embryo during gastrulation. Development $1990 ; 110$ : 521-8.

13. Campos-Ortega JA, Hartenstein V. The Embryonic Development of Drosophila melanogaster. Berlin : Springer, 1985.

14. Reynolds SD, Angerer LM, Palis J, Nasir A, Angerer RC. Early mRNAs, spatially restricted along the animal-vegetal axis of sea urchin embryos, include a protein related to tolloid and BMP-1. Development 1992 ; 114 : 769-86.

15. Wylie CC, Heasman J, Snape A, O'Driscoll M, Holwill S. Primordial germ cells of Xenopus laevis are not irreversibly determined early in development. Dev Biol $1985 ; 112$ : 66-72.

16. Blackler AW. Transfer of primordial germ-cells between two subspecies of Xenopus laevis. J Embryol Exp Morph $1962 ; 10$ : 641-51. 
17. Signoret J, Collenot A. L'organisme en développement. Des gamètes à l'embryon. Paris : Hermann, 1991.

18. Grassé PP, Devillers C. Zoologie. Tome II. Vertébrés. Paris : Masson, 1965.

19. Brien P. Biologie de la reproduction animale. Paris : Masson, 1966

20. Dunlap Pianka $\mathrm{H}$, Ctenophora. In : Giese AC, Pearse JS, eds. Reproduction of Marine Invertebrates. Vol. 1. Acoelomates and Pseudocoelomate Metazoans. New York: Academic Press, 1974 : 201-65.

21. Hyman LH. The Invertebrates: Platyhelminthes and Rhynchocoela. New York McGraw-Hill, 1951.

22. De Conninck L. Classe des nématodes In : Grassé PP, ed. Traité de zoologie. Tome IV. Fascicule II. Paris : Masson, $1965: 3-217$.

23. McGinnis W, Krumlauf R. Homeobox genes and axial patterning. Cell $1992 ; 68$. 283-302

24. Dubois R. La colonisation des ébauches gonadiques par les cellules germinales primordiales de l'embryon de poulet, en culture in vitro. J Embryol Exp Morph $1968 ; 20$ 189-213

25. Godin I, Wylie CC. TGF $\beta 1$ inhibits prolif eration and has chemotropic effect on mouse primordial cells in culture. Development $1991 ; 113: 1451-7$.

26. Shyu AB, Blumenthal T, Raff RA. A single gene encoding vitellogenin in the sea urchin. Strongylocentrotus pur puratus : sequence at the 5' end. Nucleic Acids Res 1987; 15 : 10405-17.

27. Charniaux-Cotton H. La Lignée germinale chez les Arthropodes. In : Wolff E, ed L'Origine de la lignée germinale. Paris : Hermann, 1964: 137-74.

28. Nöthiger $R$, Jonglez $M$, Leuthold $M$, Mcier-Gerschwiler P, Weber T. Sex determination in the germ line of Drosophila depends on genetic signals and inductive somatic factors. Development $1989 ; 107$ 505-18.

29. McLaren A. Relation of germ cell sex to gonadal differentiation. In : Halvorson HO, Monroy A, eds. The Origin and Evolution of Sex. New York : Liss, 1986 289-300.

30. Blackler A, Fischberg M. Transfer of primordial germ-cells in Xenopus laevis. J Embryol Exp Morphol 1961; 9 : 634-41.

31. Gallien L. Différenciation et organo genèse sexuelle des métazoaires. Paris Masson, 1973.

32. Collenot A, Gounon P, Collenot G Aspects récents de l'étude de la différenciation sexuelle des amphibiens. Étude de l'effet frec-martin chez le triton pleurodèle. Mém Soc Zool France 1977; 41 : 31-42.

$\mathrm{m} / \mathrm{s} \quad n^{\circ} \quad 6-7$ vol. 9, juin-juillet 93
33. Upadhyay S, Zamboni L. Ectopic germ cells : natural model for the study of germ cell sexual differentiation. Proc Natl Acad Sa USA 1982; 79 : 6584-8

34. Byskov AG. Regulation of initiation of meiosis in fetal gonads. Int $J$ Androl Suppl $1978 ; 2$ : 29-38.

35. Austin J, Kimble $\mathrm{J} \cdot g l p-1$ is required in the germ line for regulation of the decision between mitosis and meiosis in $C$. elegans. Cell 1987 ; 51 : 589-99.

36. Byrne BM, Gruber M, Ab G. The evolution of egg yolk proteins. Prog Biophys Molec Biol 1989 ; 53 : 33-69.

37. Trewitt PM, Heilmann LJ, Degrugillier SS, Kumaran K. The boll weevil vitellogenin gene : nucleotide sequence, structure, and evolutionary relationship to nematode and vertebrate vitellogenin genes. I Mol Evol 1992 ; $34: 478-92$

38. Junéra $\mathrm{H}$, Croisille $\mathrm{Y}$. Recherche du lieu de synthèse de la vitellogénine chez le crustacé amphipode Orchestia gammarella (Pallas). Mise en évidence d'une activation de la synthèse protéique dans le tissu adipcux sous-épidermique en liaison avec la production de vitellogénine. CR Acad Sci Paris $1980 ; 290$ : 703-6.

39. Dumont JN. Oogenesis in Xenopus lae vis Daudin VI. The route of injected tracer transport in the follicle and developing oocyte. I Exp Zool 1978 ; 204 : 193-218.

40. Nardelli D, Gerber-Huber S, van het Schip FD, Gruber M, Ab G, Wahli W. Vertebrate and nematode genes coding for yolk proteins are derived from a common ancestor. Biochemistry 1987 ; 26 : 6397-402.

41. Tétry A. Classe de sipunculiens. In Grassé PP, ed. Traité de zoologie. Tome V. Premier fascicule. Paris : Masson, 1959 785-854.

42. Dawydoff C. Classe des échiuriens. In Grassé PP, ed. Traité de zoologie. Tome V. Premier fascicule. Paris : Masson, 1959 855-907.

43. Avel M. Classe des annélides oligochètes. In : Grassé PP, ed. Traité de zoologie. Tome V. Premier fascicule. Paris : Masson, 1959 : 224-470

44. Fauvel P. Classe des annélides polychètes. In : Grassé PP, ed. Traité de zoologie. Tome V. Premier fascicule. Paris : Masson, 1959 : 95-119.

45. Goodrich ES. Studies on the Structure and Development of Vertebrates. Londres : Mac Millan, 1930.

46. Jost A. La différenciation du sexe. In : Thibault C, Levasseur MC, eds. La Reproduction chez les mammiferes et chez l'homme. Paris : INRA Ellipses 1991: 189-203.

47. Buehr M, Gu S, McLaren A. Mesonephric contribution to testis differentiation in the fetal mouse. Development $1993 ; 117$ : 273-81

48. Cate RI, Mataliano RJ, Hession C', et al. Isolation of the bovine and human genes for müllerian inhibiting substance and expression of the human gene in animal cells. Cell 1986 ; 45 : 685-98

49. Picard JY, Benarous R, Guerricr D, Josso N, Kahn A. Cloning and expression of cDNA for anti-Müllerian hormonc. Proc Natl Acad Sci USA 1986 ; 83 : 5464-8.

50. Jacob F. Le Jeu des possibles. Paris Fayard, 1981

\section{Summary}

An evolutionary interpretation of animal gametogenesis

Motility is an essential property of all animal germ cells. Gametogenesis can be divided into three main phases with respect to germ cell motility. Primordial germ cells migrate into the gonadal rudiments. A long captive phase follows, during which the germ cells differentiate into ova and spermatozoa. Mature germ cells are finally released from the gonads. In many animal groups they are brought to the exterior by genital ducts. The authors propose an evolutionary interpretation of gametogenesis. They assume that the earliest animals already had a germ line, but no gonads or sex ducts. The three phases of germ cell life then reflect three steps in the evolution of the animals' reproductive system. The first phase corresponds to an early evolutionary stage, when germ cells were not included in a gonad. The intra-gonadal phase corresponds to a later stage, when animals acquired specialized organs to harbor and protect their reproductive cells. The final stage corresponds to the most recent evolutionary innovation. Mature sex products are guided outside by specialized ducts deriving from, or connected to the excretory system.

\section{TIRÉS A PART}

H. Denis. 